\title{
HYBRID CAT SWARM OPTIMIZATION SCHEME FOR NON-PREEMPTIVE SCHEDULING OF INDEPENDENT TASK IN CLOUD COMPUTING
}

\author{
Danlami Gabi ${ }^{1}$, Abdul Samad Ismail ${ }^{2}$ and Nasiru Muhammad Dankolo ${ }^{1}$ \\ ${ }^{I}$ Department of Computer Science, Kebbi State University of Science and Technology, Aliero, Nigeria \\ ${ }^{2}$ School of Computing, Faculty of Engineering, Universiti Teknologi Malaysia
}

\begin{abstract}
The growing number of customers that are requesting computation-based-resources to meet the increasing demand of resource hungry applications have spark a greater challenge on how effective can scheduling can be carried out at the cloud datacenters. Recent advancement in the uses of metaheuristics techniques are promising approach in scheduling resources to hungry applications, but however, are limited in their performances due to issues like premature convergence. To overcome this concern with the aim to provide an effective scheduling, we propose a non-preemptive Hybrid Cat Swarm Optimization Scheme (HCSOS) to serve as an ideal solution. In the proposed scheme, orthogonal Taguchi approach is incorporated to overcome premature convergence, and minimizes local and global imbalance, while Pareto dominant strategy is used for providing customers with the option of selecting their service preferences. The results of the simulation on CloudSim tool show that our proposed scheme compared to the benchmarked schemes can achieve a minimum total execution time and cost (with up to $42.87 \%, 35.47 \%, 25.49 \%$ and $38.62 \%, 35.32 \%, 25.56 \%$ reduction). We further unveiled that a statistical analysis based on $95 \%$ confidence interval shows our proposed HCSOS scheme is remarkable in term of efficiency.
\end{abstract}

\section{KEYWORDS}

Cloud Computing, Scheduling, Cat Swarm Optimization, Pareto Dominance

\section{INTRODUCTION}

The ubiquitous cloud computing environment has no doubt become a market paradigm for resource sharing to meeting the increasing demand of resource hungry applications (Gui et al., 2014; Gabi et al., 2015). With the availability of the three cloud service models that are associated with the cloud computing, the Software as a Service (SaaS) does allow cloud customers to run their resource hungry applications remotely (Meena and Bharadi, 2016). The Plateform as a Service (PaaS) allow cost-efficient development and deployment of applications while the Infrastructure as a Service (IaaS) provide a pool of resources of varied types that can be leased by cloud customers according to their computing requirements (Zhang et al., 2017, Gabi et al., 2019). concerns on the effectiveness of the existing scheduling approaches in cloud computing. High-level research using metaheuristics techniques are now considered to be promising for executing cloud task scheduling (Zuo et al., 2015).

The Cat Swarm Optimization (CSO) is one of the metaheuristic techniques that mimics the natural behavior of Cat and has relatively proven to be better in term of convergence speed than the Particle Swarm Optimization (PSO) (Chu and Tsai, 2007). To ensure CSO become suitable for cloud scheduling, we proposed a non-preemptive HCSOS as an ideal solution. The results of the simulation of our proposed technique shows that it can provide better schedule while achieving minimum execution time and cost compared to the benchmarked schemes.

The contribution of this paper is as follows:

- Formulation of the problem and a model in relation to the problem.

- Development of an optimization scheme based on CSO, Taguchi optimization and Pareto dominance strategy.

- Implementation of the developed scheme on CloudSim Simulator tool to solve the model. 
The rest of the paper is organized as follows:

Section 2 discusses the related work. CSO optimization and the need for improvement is discussed in Section 3. Section 4 explain the Taguchi optimization and Pareto dominance strategy. Problem formulation is discussed in Section 5. Section 6 discusses the development of the task scheduling scheme. Discussion on the simulation environment is provided in Section 7. Section 8 discusses the simulation results and finally Section 9 concludes the paper.

\section{RELATED WORK}

To meet end-users Quality of Service (QoS) expectations, there is the need to ensure every computing resources (e.g. virtual machines, bandwidth, and storage) are equitably distributed on cloud tasks. Recently, metaheuristic techniques are use to solving a multi-objective task scheduling problem. Zuo et al. (2015), put forward a Multi-Objective Ant Colony Optimization (MOACO) with the objective to minimize the makespan time and customer budget cost. The simulation results showed that their proposed algorithm can achieve the optimum solution for both performance and cost. In Ramezani et al. (2013), a Multi-Objective Particle Swarm Optimization (MOPSO) algorithm is proposed. The results of the simulation show that their method can find the optimal solution in a reasonable amount of time. Liu et al. (2013) in their part developed an Improved Min-Min algorithm for cloud computing environment to provide better quality of service, dynamic priority model and cost of service) for task schedule. The results of the simulations showed that their proposed algorithms as compared with the traditional min-min algorithm showed it can increase resource utilization rate, long tasks can execute at reasonable time. In their part, Xu et al. (2015) developed a Multi-Objective Genetic Optimization Algorithm (MOGA) to minimize the average completion time, total completion time and ensure load balancing. The simulation results according to the researchers showed that their developed MOGA algorithm can produce faster convergence speed. On the other hand, Khajehvand et al. (2014) dwelt on Hybrid First-Fit Cost-Time Trade-Off (FCTT) and Workflow Planning Cost-Based (WPC) model to achieve minimum runtime and execution cost. The results of the experiment via simulation showed that proposed FCTT can reduce task runtime and allocation cost compared with MOGA and Best Effort (BE) algorithms.

Form the reviewed conducted, the CSO formed one of the promising metaheuristic algorithm that when improved can handles a muti-objective task scheduling problem. This paper seek to exploit the advantages of the CSO to make it a better optimization algorithm.

\section{CAT SWARM OPTIMIZATION AND THE NEED FOR IMPROVEMENT}

In Chue and Tsai (2007), the researchers introduced the conventional Cat Swarm Optimization (CSO) technique mimicking the behavior of natural cat. The technique operates in two modes; the resting (seeking) and chasing (tracing) mode (detail about these modes can be found in (Chu and Tsai, 2007). Although the CSO had better convergence speed than PSO (Chu and Tsai, 2007), the conventional CSO exhibit certain limitations that should possibly be address (Gabi et al., 2017). There is a possibility that its global search may not always assure superior solution when the search space increases. Converging into a stable solution is difficult due to slow convergence speed. The CSO operate independently at the global and local search level which causes velocity and position update of the conventional CSO to perform similar process and that can lead to long computation time. Thus, the need for improvement (Pradhan and Panda, 2012).

\section{TAGUCHI OPTIMIZATION AND PARETO DOMINANCE STRATEGY}

For any task schedule, the chances of locating an optimal solution that can assure minimum execution time and cost is becoming harder in a large search space like the cloud environment (Saule and Giegerich, 2015; Deb, 2001). The Taguchi method (Taguchi et al., 2000) serves as a better optimizer in achieving minimum execution time and execution cost, most especially when solving cloud task scheduling problem. The incorporation of Taguchi optimization provides better tasks mapping sequence that can reduce the task execution time. The 
pseudocode for the Taguchi optimization can be found in (Chang et al., 2015). The Pareto-Optimization is used to provide cloud customers with many non-dominant solutions as possible, by allowing sets of trade-offs between execution time and execution cost (Khajehvand et al., 2014; Liu et al., 2016). The main goal of this research is to achieve optimum execution time and execution cost on all tasks schedule on cloud resource. This study based on the definition below in solving the multi-objective task scheduling problem:

Definition 1.1 Multi-objective Optimization problem

A typical multi-objective optimization problem can be expressed as a minimization of a $K$ components of a vector function $f_{i}$ in the form (Ramezani et al., 2013):

$$
\mathbf{M f}(\vec{x})=\left[f_{1}(\vec{x}), f_{2}(\vec{x}), f_{3}(\vec{x}), \ldots \ldots f_{k}(\vec{x})\right],
$$

Where, vector of decision variable is given as $\vec{x} \forall \vec{x}=\left\{x_{1}, \ldots \ldots \ldots,\right\}$ such that $f_{i}: R^{n} \rightarrow R, i=\{1,2, \ldots ., k\}$ are the objective functions in a universe $U . \vec{f}(\vec{x})$, is the multi-objective function:

\section{PROBLEM FORMULATION}

Consider a set of independent tasks scheduled on sequence of heterogeneous virtual machines. $V=\left\{v_{k} \mid m \geq k \geq 1\right\}$, being sets of virtual machines and $m$ is the number of virtual machines. $T=\left\{t_{i} \mid n \geq i \geq 1\right\}$, represent tasks groups and $n$ is the overall number of tasks (Liu et al., 2016; Tsai et al., 2013). The goal is to dynamically assign each tasks $t_{i} \forall i=\{1,2, \ldots, n\}$ to appropriate cloud virtual machines $v_{k} \forall k=\{1,2, \ldots, m\}$, to determine the sequence as well as the timing of task executed, and the amount of cost for executing the task. The following description is assume necessary for the scheduling problem formulation: (i) Two datacentres (ii) A single service provider owns the infrastructures, where the cost of transmission is ignored; (iii) Tasks are assigned to virtual machine on FCFS basis and the total number of all possible schedules is $(n !)^{m}$ for the problem with $n$ number of task and $m$ number of virtual machine; (iv) Pre-emptive scheduling allocation policy is not allowed; (v) The cost of using a virtual machine for a time quantum varies from one to the other. Our goal is to make sure each tasks are dynamically assign to virtual machine $v_{k}$ with the right computing resource in order to find the optimum value of the total expected execution time and total expected execution cost that is incurred in executing all tasks.

Suppose $t_{i} \forall i=\{1,2, \ldots, n\}$ is scheduled on a $v_{k}$, the execution time exec $c_{k}$ of all tasks processed on a $v_{k}$ is computed using Equation 2 (Ramezani et al., 2013). The total execution time Texe $e_{k}$ of all tasks $t_{i}$ processed on all virtual machines $v_{k} \forall k=\{1,2, \ldots, m\}$ is computed using Equation 3 (Ramezani et al., 2015).

$$
\begin{aligned}
\text { exec }_{k} & =\sum_{i=1}^{n} x_{i k} * \frac{t_{i}}{n p e_{k} \times v_{\text {kmips }}}, \\
\text { Texec }_{k} & =\sum_{k=1}^{m} \sum_{i=1}^{n} x_{i k} * \frac{t_{i}}{n p e_{k} \times v_{\mathrm{kmips}}}
\end{aligned}
$$

Where, exec $_{k}$ is the execution time of running tasks on one virtual machine; $x_{i k}$ is equal to one 1 if task $t_{i}$ is assign to $v_{k}$ otherwise, $x_{i k}=0 ; t_{i}$ is the task whose length is given in Millions Instructions (MI); $v_{k m i p s}$ is the $v_{k}$ speed whose unit is in Million Instructions Per Second (MIPS); $n p e_{k}$ is the number of processing element of a virtual machine $v_{k}$. The execution cost model is a multi-objective model that incorporates the execution time model shown in Equation 4. Assuming the cost of executing tasks on a virtual machine per hour (hr) is known, Equation 4 hold for the cost exe cost $_{k}$ to exeute tasks $t_{i}$ on a virtual machine $v_{k}$ per time quantum in second (Ramezani et al., 2015).

exe $_{\text {cost }_{k}}=\frac{1}{3600} \sum_{i=1}^{n} v_{\text {kcost }} * x_{i k} * \frac{t_{i}}{n p e_{k} \text { VV }_{\text {mips }}}$,

Where, $v_{k c o s t}$ is the monetary cost of one unit $v_{k}$ per hour, in US dollar.

Such that: $x_{i k}=\left\{\begin{array}{l}1, \text { if task } i \text { is assign on } v_{k} \\ 0, \text { otherwise }\end{array}\right.$

When more than one $v_{k} \forall k=\{1,2, \ldots, m\}$ are used by a service provider to execute many tasks, the total tasks execution cost TTexe $_{\text {cost }_{k}}$ by all virtual machine in a datacenter in a datacenter can be computed using Equation (6). 
TTexe $_{\text {cost }_{k}}=\frac{1}{3600} \sum_{k=1}^{m} \sum_{i=1}^{n}$ vcost $_{k} * x_{i k} * \frac{t_{i}}{n p e_{k} V_{\text {mips }_{k}}}$

The multi-objective task scheduling mathematical model can be expressed as follow:

$\operatorname{Min} \mathrm{F}(\mathrm{X})=\left[\operatorname{Texec}_{k}(X)\right.$, TTexe $\left._{\text {cost }_{k}}(X)\right]$

Subject to: $\sum_{k=1} x_{i k}=1, \forall i=1,2, \ldots \ldots, n ; \quad x_{i k} \in\{0,1\}, \forall i, k$

Eqn.8 is the multi-objective optimization model to be solved by applying our proposed multi-objective scheme.

\section{THE PROPOSED TASK SCHEDULING SCHEME}

The proposed HCSOS scheme consists of two phases (global and local search) that combined and solved the optimization problem. Each cat symbolizes the choice of virtual machine used for the task schedule. This is encoded in $[1 \times \mathrm{n}]$ vector, with $n$ belonging to a number of tasks. We assumed that each virtual machine in a datacenter has different cost per time quantum. Every cat has dimension $D$ with $n$ tasks assigned and the model associated with each cat are based on two objective function; the total execution time $\left(\operatorname{Texec}_{k}(X)\right)$ and total execution $\operatorname{cost}\left(\right.$ TTexe $\left._{\text {cost }_{k}}(X)\right)$. When a cat traverses all tasks, it formed a feasible solution to the problem. Each cat has both position and velocity vector. The position of the cat symbolizes the solution that is attained by the cat. A Mixed Ratio (MR) is used to specify two group of cats (seeking and tracing). The cats are moved into either seeking or tracing mode at random using the value specified by the $M R$. When the cats reach their desired target, their fitness value are computed based on the defined objective of the scheduling problem $\left(\right.$ Texec $_{k}$ and TTexe cost $_{k}$ ). To update the velocity, the best velocity among the two velocity sets that returns an optimal solution for each run is selected if and only if the condition specified in Equation 8 hold.

$$
\vec{V}_{k, d}\left\{\begin{array}{l}
\max v, \text { if }\left[\vec{V}_{k, d}+\overrightarrow{V o}_{k, d}(t)\right]>\text { maximum velocity, } \\
\vec{V}_{k, d}+\overrightarrow{V o}_{k d}(t) \text { otherwise }
\end{array}\right.
$$

Where, $\max v$ is the maximum velocity attain by the cat; $\vec{V}_{k, d}$ is the velocity of the cats; $\overrightarrow{V o}_{k, d}(t)$ is the candidate solutions. A dominant strategy is used to compare the optimum solution and is stored at the archive, and the final velocity that should formulate the latest velocity is selected. This velocity returns optimal solution which is used to compute the new position of the cat as indicated in Equation 9.

$$
\vec{X}_{k, d}=\vec{X}_{k, d}+\vec{V}_{k, d}
$$

Where, $\vec{X}_{k, d}$ is the position of the Cat; $\vec{V}_{k, d}$ is the velocity attain by the cat. In order to ensure the quality of the solution, avoid being trapped at the local optima, the quality of the feasible solution is therefore evaluated using the fitness function. This fitness function is set based on an optimization model of the scheduling problem. As earlier reported, the optimal solution of each cat is stored at the archive. Every cat is assessed with a value of fitness functions and all Pareto optimal solutions that are stored at the archive. Hence, the fitness function $\operatorname{QoS}(\vec{X})$ using Equation 10 was used for the evaluation:

$\operatorname{QoS}(\vec{X})=\sum_{j=1}^{m} W_{j} f_{j}\left(\overrightarrow{X_{l}}\right),\left\{\forall \overrightarrow{X_{l}} \in\right.$ Archieve $\}$

Where, $m$ is the number of objective functions and $W_{j}$ is the preference weight for every objective function $\left(f_{j}\left(\vec{X}_{\imath}\right)\right)$. Algorithm 1 provides the pseudocode for the proposed HCSOS task scheduling algorithm. 


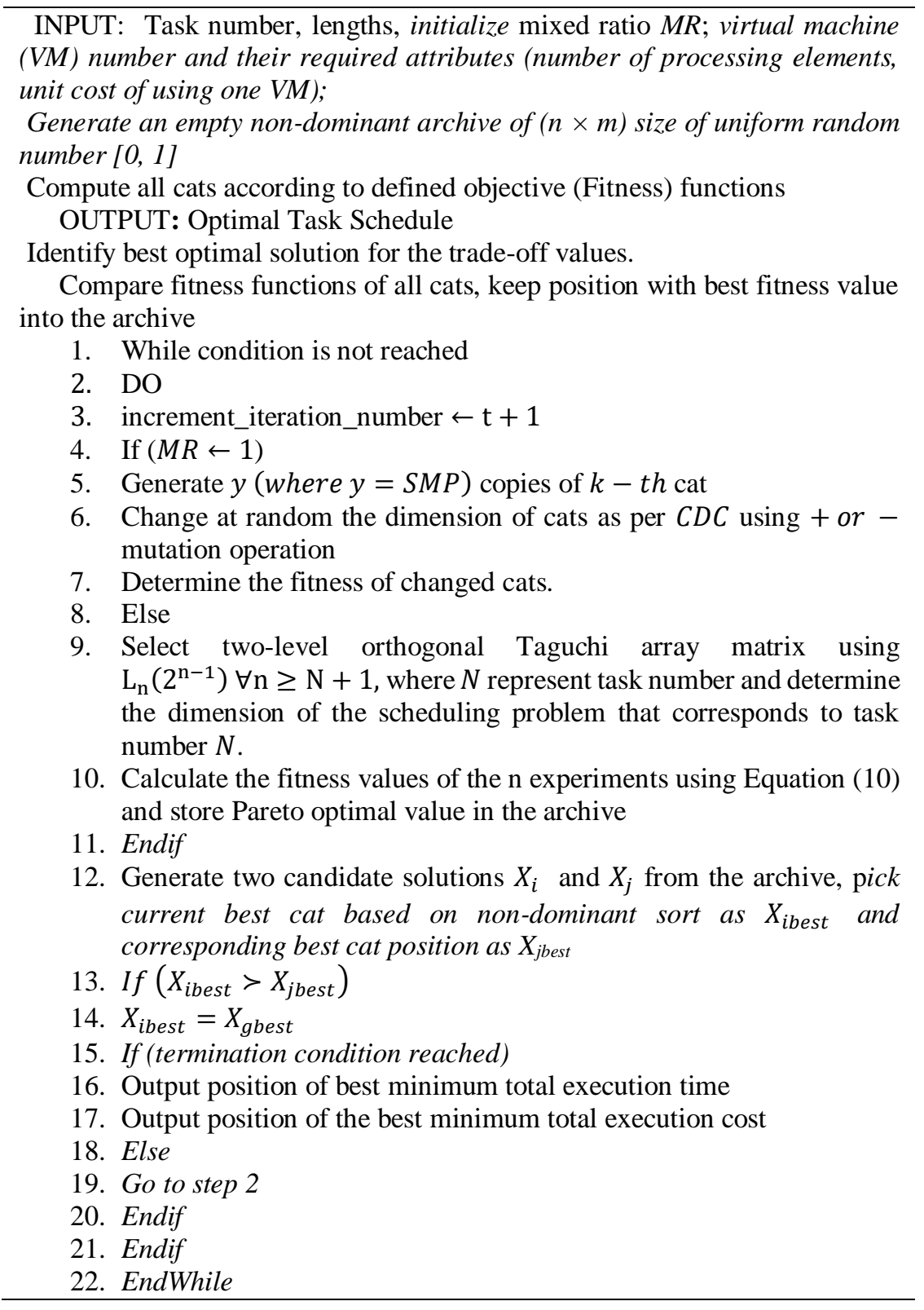

Algorithm 1. HCSOS-Based Algorithm

\section{SIMULATION ENVIRONMNET}

CloudSim 3.0.3 (Calheiros et al., 2010) was installed on Eclipse-java-luna-SR2-win32-x86-64 as the simulation environment. The proposed DMOOTC task scheduling scheme was compared with that of Multi-Objective Particle Swarm Optimization (MOPSO) (Ramezani et al., 2015), Multi-Objective Ant Colony Optimization (MOACO) (Zuo et al., 2015) and Min-Min (Liu et al., 2013) task scheduling schemes. The choices of properties for the datacenter host, task and virtual machines were based on (Gabi et al., 2018). The estimated cost $(0.17 \$+0.05 \$=0.22 \$ / \mathrm{hr})$ of using a unit virtual machine over a time quantum while values for inertia weight and that of coefficient factors $\left(c_{1} c_{2}\right)$ for MOACO, MOPSO, and DMOOTC were specified as used in (Elberhart and Shi, 2000). The parameter settings for the scheduling algorithms are; MOPSO (Particle size:100, Self-recognition coefficients $\left(c_{1} c_{2}\right): 2.0$, Uniform random number $\left(R_{1}\right)$ : [0,1], Maximum 
iteration:1000, Inertia weight $(W)$ : 90-40\%, Mixed ratio:2\%); DMOOTC (Cat size:100, Count Dimension to Change: $5 \%$, Self-recognition coefficients $\left(c_{1}\right)$ : 2.0, Uniform random number $\left(R_{1}\right)$ : [0,1], Maximum iteration:1000, Inertia weight $(W): 90-40 \%$, Mixed ratio: 2\%); MOACO (Pheromone persistence $\alpha: 0.3$, Importance of pheromone $(\gamma): 1$, Importance of resource innate attribute $(\beta): 1$, Pheromone evaporation value $(\rho)$ : 0.3, Iteration number: 1000, Number of ant $m: 100)$.

\section{RESULTS AND DISCUSSION}

The simulation results are discuss in this section. Task instances from 20 to 100 with 20 heterogeneous virtual machines were used. Ten (10) independent simulation runs were conducted that reveals the efficiency of the proposed scheme. Table 1 show the results obtained based on average value of the simulation runs in term of execution time and execution cost while Table 2 show the improvement gain by the proposed HCSOS task scheduling scheme compared to the benchmarked schemes. In Table 1 precisely, Min-Min scheduling scheme show better performance in term of time and cost at the task scheduling intervals of 20 to 50 compared to MOACO, MOPSO and the proposed HCSOS. As the task scheduling intervals changes over time (see 70 to 100 task instances), its performance degrades to an unprecedented level. The MOACO task scheduling scheme on the other hand, performed better when tasks are schedule from 20 to 40, its performance suddenly degrade with increase in tasks size. This can be attributed to the traversing process of the ant colony approach at the local region that becomes entrapped at the local optima. In term of the MOPSO task scheduling scheme, better performance is achieved when 50 to 100 tasks are scheduled on virtual machine compared to MOACO and Min-Min scheduling schemes. The results in Table 2 show the performance improvement gained from the three benchmarked schemes. In the overall performance, our proposed DMOOTC task scheduling scheme is able to minimized the total tasks execution time with $42.87 \%, 35.47 \%$ and $25.49 \%$ compared to Min-Min, MOACO and MOPSO task scheduling schemes.

To further elaborate on the performance of our proposed scheme, we show a conputed $95 \%$ confidence intervals in Table 3. The computed value was derived using Equation 11 (Hosmer and Lemeshow, 1992).

Confidential Interval $(\mathrm{CI})=\bar{x} \pm t \frac{s}{\sqrt{n}}$,

where, $\bar{x}$ is the mean; $t$ represent the $\mathrm{t}$-distribution that is derived from the $\mathrm{t}$ distribution table; $s$ is the standard deviation of the sample data derived after running the task instances on a virtual machine and $n$ represent the number of samples. The confidence interval help provide how significance our obtained results. The smaller the value of the confidential intervals as compared to the benchmarked algorithms the more precise our estimate. The statistical analysis indicated that for $95 \%$ confidentce intervals obtained for all the scheduling scheme, the computed value of the HCSOS is less compared to that of the benchmarked schemes. Although the smaller estimate of the $\mathrm{CI}$ is an indication of a significant performance in term of execution time, it can be concluded that the HCSOS scheduling scheme has outperformed the benchmarked schemes by providing minimum execution time.

It can be concluded that, the overal performance shown by our proposed HCSOS task scheduling scheme is as a result of the incorporation of the Taguchi Orthogonal approach and Pareto dominance strategy at the local search of the CSO.

Table 1. Average results on the computed execution time-cost

\begin{tabular}{ccccccccc}
\hline Task & \multicolumn{2}{c}{ Improved Min-Min } & \multicolumn{2}{c}{ MOACO } & \multicolumn{2}{c}{ MOPSO } & \multicolumn{2}{c}{ HCSOS } \\
\cline { 2 - 8 } & $\begin{array}{c}\text { Execution } \\
\text { time }(\mathrm{s})\end{array}$ & $\begin{array}{c}\text { Execution } \\
\text { cost }(\$) / \mathrm{hr}\end{array}$ & $\begin{array}{c}\text { Execution } \\
\text { time }(\mathrm{s})\end{array}$ & $\begin{array}{c}\text { Execution } \\
\text { cost }(\$) / \mathrm{hr}\end{array}$ & $\begin{array}{c}\text { Execution } \\
\text { time }(\mathrm{s})\end{array}$ & $\begin{array}{c}\text { Execution } \\
\text { cost }(\$) / \mathrm{hr}\end{array}$ & $\begin{array}{c}\text { Execution } \\
\text { time }(\mathrm{s})\end{array}$ & $\begin{array}{c}\text { Execution } \\
\text { cost }(\$) / \mathrm{hr}\end{array}$ \\
\hline 20 & 21.46 & 4.72 & 27.54 & 6.05 & 26.86 & 5.90 & 25.40 & 5.47 \\
30 & 39.16 & 8.61 & 56.32 & 12.39 & 70.29 & 15.45 & 53.58 & 11.78 \\
40 & 60.44 & 13.29 & 87.56 & 19.26 & 92.29 & 20.59 & 53.92 & 11.86 \\
50 & 66.62 & 14.65 & 163.49 & 35.96 & 147.28 & 32.40 & 129.19 & 28.42 \\
60 & 209.82 & 46.15 & 243.89 & 53.65 & 206.50 & 45.43 & 162.77 & 35.80 \\
70 & 318.81 & 70.13 & 312.78 & 68.10 & 287.21 & 63.18 & 240.12 & 52.82 \\
80 & 505.02 & 111.10 & 407.54 & 89.65 & 334.99 & 73.69 & 312.28 & 68.70 \\
90 & 768.78 & 169.13 & 674.89 & 148.47 & 538.09 & 118.37 & 331.62 & 72.94 \\
100 & 1096.40 & 241.21 & 754.90 & 166.07 & 663.34 & 145.93 & 454.52 & 99.98 \\
\hline
\end{tabular}


Table 2. Performance Improvement Rate (PIR\%)

\begin{tabular}{lcccc}
\hline & Improved Min-Min & MOACO & MOPSO & HCSOS \\
\hline Total execution time & 3086.51 & 2732.94 & 2366.85 & 1763.40 \\
PIR (\%) over Improved Min-Min & & 11.455 & 23.31 & $\mathbf{4 2 . 8 7}$ \\
PIR (\%) over MOACO & & & 13.39 & $\mathbf{3 5 . 4 7}$ \\
PIR (\%) over MOPSO & & & $\mathbf{2 5 . 4 9}$ \\
\hline
\end{tabular}

Table 3. Significance on confidential interval

\begin{tabular}{lcccc}
\hline & $\begin{array}{c}\text { Improved } \\
\text { Min-Min }\end{array}$ & MOACO & MOPSO & HCSOS \\
\hline Degree of freedom & 9 & 9 & 9 & 9 \\
Confidence level & 0.025 & 0.025 & 0.025 & 0.025 \\
t-distribution & 2.262 & 2.267 & 2.267 & 2.262 \\
Mean & 342.94 & 303.21 & 262.98 & 195.93 \\
Standard Deviation & 378.38 & 264.49 & 218.04 & 148.25 \\
Lower bound & 72.28 & 114.02 & 107.01 & 90.04 \\
Upper bound & 613.60 & 492.40 & 418.95 & 254.14 \\
Confidential Interval & $(72.28,613.60)$ & $(114.02,492.40)$ & $(107.01,418.95)$ & $(90.04,254.14)$ \\
\hline
\end{tabular}

\section{CONCLUSION}

In this paper, the efficiency of our developed scheme has been revealed using simulation. An average of 10 simulation runs for all scheduled task on virtual machines was computed. The results of the simulation obtained from our proposed HCSOS shows minimum time and cost of execution for all scheduled task on virtual machines compared to that of the benchmarked schemes. The used of Taguchi Orthogonal approach and Pareto-dominance strategy has improved the local search procedure of the CSO that mitigates premature convergence. To further reveal how significant the performance of our developed HCSOS, a statistical significance was reported which shows better results. Further study is therefore necessary to investigate the scalability of the proposed scheme using large computing workloads.

\section{REFERENCES}

Calheiros R. N. et al, 2010. CloudSim: A toolkit for modeling and simulation of cloud computing environments and evaluation of resource provisioning algorithms. Software - Practice and Experience, Vol. 41, No. 1, pp 23-50.

Chang H.-C. et al., 2015. Solving the Flexible Job Shop Scheduling Problem with Makespan Optimization by Using a Hybrid Taguchi-Genetic Algorithm. IEEE Journals and Magazines, Vol.3, No. 1, pp 1740-1754.

Chu S.-C. and Tsai P.-W. 2007. Computational intelligence based on the behavior of cats. International Journal of Innovative Computing, Information and Control, Vol. 3, No. 1, pp 163-173.

Eberhart, R. C., Shi, Y. 2000. Comparing Inertia Weights and Constriction factors in particle swarm optimization. Proceedings of the IEEE Conference on Evolutionary Computation (ICEC). La Jolla, CA, USA, USA., DOI: 10.1109/CEC.2000.870279.

Gabi D. et al. 2019. Minimized Makespan Based Improved Cat Swarm Optimization for Efficient Task Scheduling in Cloud Datacenter. Proceedings of the 2019 3rd High Performance Computing and Cluster Technologies Conference (HPCCT 2019). Guangzhou, China, pp. 16-20.

Gabi D. et al, 2018. Hybrid cat swarm optimization and simulated annealing for dynamic task scheduling on cloud computing environment. Journal of Information and Communication Technology, Vol.17, Vol. 3, pp 435-467.

Gabi D. et al. 2017. Scalability-Aware scheduling optimization algorithm for multi-objective cloud task scheduling problem. Proceedings of the 2017 6th ICT International Student Project Conference (ICT-ISPC). Skudai, Malaysia, pp. 1-6.

D. et al. 2015.Systematic Review on Existing Load Balancing Techniques in Cloud Computing. International Journal of Computer Applications, Vol.125, No.9, pp. 16-24. 
Gui Z et al, 2014. A Service Brokering and Recommendation Mechanism for Better Selecting Cloud Services. PLoS ONE, Vol. 9, No. 8, pp 1-20.

Hosmer D. W. and Lemeshow S. 1992. Confidence Interval Estimation of Interaction. Epidemiology, Vol. 3, No. 5, pp 452-456.

Khajehvand V. et al, 2014. Multi-objective and Scalable Heuristic Algorithm for Workflow Task Scheduling in Utility Grids. Journal of Optimization in Industrial Engineering, Vol. 14, No.1, pp 27-36.

Liu G. et al, 2013. An Improved Min-Min Algorithm in Cloud Computing. In: Du Z. (eds) Proceedings of the 2012 International Conference of Modern Computer Science and Applications. Advances in Intelligent Systems and Computing, Vol 191. Springer, Berlin, Heidelberg, pp 47-52.

Liu J. et al, 2016. Multi-objective scheduling of Scientific Workflows in multisite clouds. Future Generation Computer Systems, Vol. 63, No. 1, pp 76-95.

M. and Bharadi V. A. 2016. Performance Analysis of Cloud Based Software as a Service (SaaS) Model on Public and Hybrid cloud. Proceedings of the 2016 Symposium on Colossal Data Analysis and Networking (CDAN). Indore, Madhya Pradesh, pp 18-19.

Pradhan P.M. and Panda G. 2012. Solving Multi-objective problems using cat swarm optimization. An international Journal of Expert System with Application, Vol. 39, No. 1, pp 2956-2964.

Ramezani F. et al, 2013. Task Scheduling Optimization in Cloud Computing Applying Multi-Objective Particle Swarm. LNCS 8274, pp 237-251, Springer-Verlag Berlin Heidelberg.

Taguchi G. et al, 2000. Robust Engineering: Learn how to boast quality while reducing cost and time to work. New York: McGraw-Hill.

Tsai J.-T. et al, 2013. Optimized Tasks Scheduling and Resource Allocation on Cloud Computing Environment using Improved Differential Evolution Algorithm. Computers and Operations Research, Vol. 40, No. 1, pp 3045-3055.

Xu Z. et al, 2015. Task Scheduling Based on Multi-Objective Genetic Algorithm in Cloud Computing. Journal of Information and Computational Science, Vol. 12, No. 4, pp 1429-1438.

Zhang Y. et al., 2017. Agent and Cyber-Physical System Based Self-Organizing and Self-Adaptive Intelligent Shopfloor. IEEE Transaction on Industrial Informatics, Vol. 13, No. 2, pp 737-747.

Zuo L. et al, 2015. A Multi-Objective Optimization Scheduling Method Based on the Ant Colony Algorithm in Cloud Computing. IEEE Access: The journal for rapid open access publishing, Vol. 3, No. 1, pp 2687-2699. 